\title{
NISTIR 6051
}

\section{Narrow Bandwidth Grating-Assisted Acousto-Optic Tunable Filter}

\section{Eung Gi Paek}

Information Technology Laboratory

National Institute of Standards and Technology*

Gaithersburg, MD 20899

\section{Joon Y. Choe}

Tae K. Oh

Naval Surface Warfare Center

Dahlgren, VA 22192

*U.S. DEPARTMENT OF COMMERCE

Technology Administration

National Institute of Standards and Technology

Gaithersburg, MD 20899-0001

$Q C$ 



\section{NISTIR 6015}

\section{Narrow Bandwidth Grating-Assisted Acousto-Optic Tunable Filter}

\section{Eung Gi Paek}

Information Technology Laboratory

National Institute of Standards and Technology* Gaithersburg, MD 20899

\section{Joon Y. Choe \\ Tae K. Oh}

Naval Surface Warfare Center

Dahlgren, VA 22192

*U.S. DEPARTMENT OF COMMERCE

Technology Administration

National Institute of Standards and Technology

Gaithersburg, MD 20899-0001

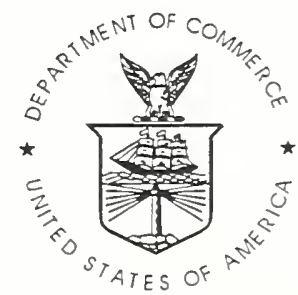

U.S. DEPARTMENT OF COMMERCE

William M. Daley, Secretary

TECHNOLOGY ADMINISTRATION

Gary R. Bachula, Acting Under Secretary for Technology 


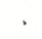




\title{
Narrow Bandwidth Grating-Assisted Acousto-Optic Tunable Filter
}

\author{
Eung Gi Paek \\ Information Technology Laboratory \\ National Institute of Standards and Technology, Gaithersburg, MD 20899 \\ Joon Y. Choe and Tae K. Oh \\ Naval Surface Warfare Center, Dahlgren, VA
}

Abstract - An experimental acousto-optic tunable filter that has a narrow spectral bandwidth $(0.2$ $\mathrm{nm}$ at $1550 \mathrm{~nm})$ and a fast $(10 \mu \mathrm{sec})$ tuning capability with a continuous tuning range of $52 \mathrm{~nm}$ is described. By combining a conventional diffraction grating with an acousto-optic beam deflector, spectral bandwidth has been significantly reduced and the number of usable wavelength channels within the erbium-doped fiber amplifier (EDFA) passband is increased by approximately 20 times. The filter also permits simultaneous and independent selection/routing of many wavelength channels for multi-channel wavelength division multiplexing (WDM) filters/routers/switches or fast scan optical spectrum analyses.

To be submitted to Applied Physics Letters 


\section{Introduction}

Recent interest in dense wavelength-division multiplexing (WDM) increasingly demands a faster tunable spectral filter with a narrower bandwidth to permit a larger number of wavelength channels within the EDFA passband $(1.53 \mu \mathrm{m}$ to $1.57 \mu \mathrm{m})$ [1-4]. Acousto-optic devices are promising for this application because they provide fast wavelength tuning (on the order of a few microseconds), wide tuning range $(>100 \mathrm{~nm})$ and simultaneous and independent selection of many wavelengths [5-13]. However, conventional acousto-optic tunable filters (AOTF's) have the sidelobe crosstalk problem that seriously limits their use in current dense WDM. Most of the suggested methods to reduce the problem have been limited to apodization, tapering, etc. $[14,15]$

In this paper, we propose a different approach to reduce the crosstalk significantly by reducing the spectral bandwidth with the assistance of a diffraction grating. Although gratingassisted coupling of waveguides has been used for many years to implement directional couplers, filters, lasers, and other planar devices [16-18], one should note that the grating vector in our device is normal to the light propagation direction, unlike other conventional grating-assisted devices in which grating vector is along the light propagation direction. We use the concept to resolve the crosstalk problem of AOTF's for the first time to our knowledge. As will be explained later, By selecting a slow shear mode $\mathrm{TeO}_{2}$ crystal $(30 \mathrm{MHz}-50 \mathrm{MHz})$ with a $10 \mu \mathrm{s}$ aperture size, and by incorporating it with a 1200 grooves/mm diffraction grating, high spectral resolution $(<0.2 \mathrm{~nm})$, broad tuning range ( $50 \mathrm{~nm}$ to cover the entire EFDA passband) and fast tuning speed $(<10 \mu \mathrm{s})$ are obtained in the $1.55 \mu \mathrm{m}$ region. 
Figure 1 compares three types of acousto-optic devices that have wavelength-selection capability : (a) acousto-optic tunable filters (AOTF's) (b) conventional acousto-optic spectrum analyzers (AOS's) and (c) our novel grating-assisted acousto-optic tunable filter (GA-AOTF) proposed in this paper. Table I also compares the three devices with regard to the grating period or the beat length, the number of gratings (or beats in case of birefringent AOTF's) for the given interaction length $(\mathrm{L})$, spectral bandwidth and tuning range.

AOTFs (shown in Figure 1(a)) normally have a collinear configuration using birefringent acousto-optic materials [5-13] and are most commonly used in current WDM applications due to its compact configuration and potential for low power consumption in an integrated device. The spectral bandwidth of an AOTF is given by

$$
\delta \lambda=\frac{\lambda}{\mathrm{N}}, \quad \mathrm{N}=\frac{|\Delta \mathrm{n}| \cdot \mathrm{L}}{\lambda},
$$

where $\Delta \mathrm{n}$ is the birefringence inside an acousto-optic medium, $\mathrm{L}$ is acousto-optic interaction length, $\lambda$ is the wavelength of light and $\mathrm{N}$ is the number of beats within the interaction length. Since the birefringence is small $(\Delta \mathrm{n}<<1), N$ is small and thus the bandwidth is relatively large compared with conventional diffraction gratings. For a typical example of $\Delta \mathrm{n}=0.08, \mathrm{~L}=2 \mathrm{~cm}$, and $\lambda=1550 \mathrm{~nm}, \delta \lambda=1.5 \mathrm{~nm}$ is obtained [13]. The sidelobes due to the relatively large bandwidth cause a crosstalk problem in dense WDM.

In an acousto-optic spectrum analyzer shown in Figure 1 (b), spectral bandwidth is given by

$$
\delta \lambda=\frac{\lambda}{\mathrm{N}}, \quad \mathrm{N}=\frac{\mathrm{L}}{\Lambda_{\mathrm{AO}}},
$$


where $\Lambda_{\mathrm{AO}}$ is the period the acoustic wave inside a medium and $\mathrm{N}$ is the number of acoustic periods within the optical aperture and is equal to time-bandwidth product (TBP). The TBP can be increased by increasing the frequency of the sound, choosing a "slow" AO material, and by increasing the optical aperture size. However, all these three parameters are related with each other. For instance, the increase in frequency causes an acoustic attenuation which is proportional to frequency squared which in turn results in a reduction in a usable aperture size. Also, the use of a slow AO material will result in slower tuning speed. In addition, the acoustic material should have high figure of merits to permit high diffraction efficiency at low power consumption. Typically, TBP of an acousto-optic beam deflector (AOBD) is limited to less than 1,000 in the $1.55 \mu \mathrm{m}$ wavelength region, yielding a broad bandwidth. Furthermore, the small diffraction angle of an $\mathrm{AOBD}$ due to the large acoustic grating period $\Lambda_{\mathrm{AO}}$ requires a lens with a long focal length in order to separate the output channels, making a tunable filter bulky. Therefore, it is known that an acousto-optic beam deflector followed by a slit or pinhole is not attractive as a tunable filter [4].

The above-mentioned bandwidth limitation of an acousto-optic tunable filter can be significantly alleviated by simply adding a conventional diffraction grating after an $\mathrm{AOBD}$, as shown in Figure 1 (c). In this case, beam deflection angle, $\Delta \theta$, for an increment in wavelength, $\Delta \lambda$, and an increment in acoustic frequency, $\Delta \mathrm{f}$, is given by

$$
\Delta \theta=\frac{\Delta \lambda}{\mathrm{d}}+\frac{\mathrm{f} \cdot \Delta \lambda}{\mathrm{v}}+\frac{\lambda \cdot \Delta \mathrm{f}}{\mathrm{v}}
$$

where, $\lambda, d, v$, and $f$ are light wavelength, the period of a diffraction grating, acoustic velocity, and the center frequency of an $\mathrm{AOBD}$, respectively. In general, $\mathrm{d}<<\Lambda_{\mathrm{AO}}=\mathrm{V} / \mathrm{f}$ and so the beam deflection angle can be approximated to 


$$
\Delta \theta \approx \frac{\Delta \lambda}{\mathrm{d}}+\frac{\lambda \cdot \Delta \mathrm{f}}{\mathrm{v}}
$$

Therefore, wavelength-dependent dispersion is mainly determined by a diffraction grating (first term) and an AOBD functions as a beam steerer to shift the whole spectrum up and down in the spectrum plane. Desired wavelengths can be selected through a pinhole, a slit or an optical fiber in the spectrum plane by adjusting if frequencies. Spectral bandwidth, $\delta \lambda$, is given by

$$
\delta \lambda=\frac{\lambda}{N}, \mathrm{~N}=\mathrm{L} / \mathrm{d}
$$

where $\mathrm{N}$ is the number of grating periods within the optical aperture, L. Here one should note that the short periods, $d$, of conventional diffraction gratings (approximately $1 / 20$ times of those of acousto-optic beam deflectors) permit large $\mathrm{N}$ and thus narrow spectral bandwidths. Tuning range, $\Delta \lambda_{\mathrm{T}}$ can be calculated by equating the first and second term of Eq. (4) to yield

$$
\Delta \lambda_{\mathrm{T}}=\lambda \cdot \mathrm{d} \cdot \Delta \mathrm{f} / \mathrm{v}=\lambda \cdot \frac{\Delta \mathrm{f}}{\mathrm{f}} \cdot \frac{\mathrm{d}}{\Lambda_{\mathrm{AO}}}
$$

For a typical example of a slow shear mode $\mathrm{TeO}_{2} \mathrm{AOBD}$ at $1.55 \mu \mathrm{m}(\mathrm{v}=617 \mathrm{~m} / \mathrm{s}, \mathrm{f}=35 \mathrm{MHz}$, $\Delta \mathrm{f}=25 \mathrm{MHz})$ and a diffraction grating (1200 grooves $/ \mathrm{mm}), \mathrm{d}=1 / 1200 \mathrm{~mm}=0.83 \mu \mathrm{m}, \Lambda_{\mathrm{AO}}=$ $\mathrm{v} / \mathrm{f}=617 \mathrm{~m} / \mathrm{s} / 35 \mathrm{MHz}=17.6 \mu \mathrm{m}$. Therefore, spectral bandwidth $\delta \lambda=0.2 \mathrm{~nm}$ and tuning range $\Delta \lambda_{\mathrm{T}}=52 \mathrm{~nm}$. Here one should note that the tuning range has been reduced by the ratio, $\mathrm{d} / \Lambda_{\mathrm{AO}}$ $(=5 \%)$ compared with that of the original AOS (shown in Figure 1(b)). This is a penalty paid for the narrow spectral bandwidth. However, the reduced tuning range is still broad enough to cover the whole EDFA passband which is the main interest in current WDM. For applications requiring a larger tuning range, an acousto-optic material with a larger $\Delta \mathrm{f} / \mathrm{v}$ ratio can be used. As in other acousto-optic devices, many rf frequencies can be applied to the AOBD for simultaneous and 
independent selection of many different channels through the same output node. Also, unlike the AOTF, the outputs can be directly routed to many different nodes.

\section{EXPERIMENTAL SETUP}

Figure 2 shows a schematic of our experimental setup to prove the concept of the narrow bandwidth GA-AOTF. The light from a light source (1.55 $\mu \mathrm{m}$ wavelength region) is coupled into a single-mode fiber and is divided into two by a directional coupler : one goes to a conventional optical spectrum analyzer (ANDO AQ-6315A) and the other goes to our GA-AOTF. Light from the single-mode fiber which is pigtailed to the light source is collimated by a lens $L_{1}(f=5 \mathrm{~cm}$, $\mathrm{D}=1.8 \mathrm{~cm}$ ) and is deflected by an AOBD. The AOBD we used in our experiment was originally designed for visible wavelengths (Crystal Technology, Model 4050-2A, $\mathrm{TeO}_{2}$, slow shear mode, $514 \mathrm{~nm}$ ) and was not optimized at $1.55 \mu \mathrm{m}$. However, it showed a reasonably high efficiency (around $10 \%$ at $1550 \mathrm{~nm}$ region) at $\mathrm{rf}$ frequencies of $30 \mathrm{MHz}$ to $50 \mathrm{MHz}$. A diffraction grating (Richardson Grating Lab., 1200 grooves $/ \mathrm{mm}, 1.55 \mu \mathrm{m}$, Littrow angle $=68.41^{\circ}$, gold coated) diffracts the light from the AOBD. Incident angle to the diffraction grating is close to the normal to the surface and the diffraction angle is around $30^{\circ}$. The diffracted light is folded by an IR mirror and is focused by a lens (focal length $=38 \mathrm{~cm}$ ) at the focal plane. The spectrum thus obtained at the focal plane is detected by an IR camera (Hamamatsu, Model No. C2741). A special care is taken to ensure high collimation by using a plane parallel plate.

\section{Experimental results}


Figure 3 shows the experimental results obtained from the setup described above. As a test light source, a $1.55 \mu \mathrm{m}$ fiber-pigtailed Faby-Perot laser diode is used. The Fabry-Perot laser diode has many longitudinal modes separated by $1.2 \mathrm{~nm}$ which is beyond the resolution of commercially available AOTFs. Figure 3 (a) shows a spectrum obtained from a conventional AOS shown in Figure 2 (b). As expected, it cannot resolve the Fabry-Perot lines. Figure 3 (b) shows a spectrum obtained by the GA-AOTF in which a diffracting grating is inserted to the system in Figure 2 (b). As shown in the figure, the Fabry-Perot lines are clearly resolved, even though the IR camera is blurred due to over-exposure.

Figure 4 shows the tuning capability of the GA-AOTF. The whole spectrum can be shifted rapidly (within $10 \mu \mathrm{s}$ ) along the acoustic wave direction by varying the acoustic frequencies; (a) $40 \mathrm{MHz}$, (b) $45 \mathrm{MHz}$ and (c) $55 \mathrm{MHz}$. Therefore, through a pinhole, slit or a fiber in this plane, desired wavelengths can be selected within several microseconds.

To keep the access time less than $10 \mu \mathrm{s}$, the aperture of the AOBD is reduced to about 6 $\mathrm{mm}$, resulting in the spectral bandwidth of $0.2 \mathrm{~nm}$. The bandwidth can be further reduced by several different ways: either by increasing the aperture size of the AOBD by sacrificing access time, or by using faster acousto-optic materials such as $\mathrm{LiNbO}_{3}$.

\section{Conclusion:}

We have proposed and demonstrated a novel GA-AOTF that has a narrow spectral bandwidth to permit a significantly (around 20 times) larger number of wavelength channels 
compared with conventional AOS's or the AOTF's, while preserving fast tuning speed (on the order of $\mu \mathrm{s}$ ) and a broad tuning range to cover the whole EDFA passband. The filter also permits simultaneous and independent selection/routing of many wavelength channels and thus can be used as multi-channel wavelength division multiplexing (WDM) filters/routers/switches or fast scan optical spectrum analyzers[19]. The proposed device architecture can be integrated into a waveguide device for low power consumption and a compact size using the technique developed by Soole et al [20].

Acknowledgment: This work was supported by the U.S. Office of Naval Research (ONR) under contract NC0014-97-F-0021. 


\section{References}

1. N.K. Cheung, K. Nosu, and G. Winzer, eds., "Special issue on dense wavelength division multiplexing for high capacity and multiple access communications systems," IEEE Jour. Sel. Areas in Commun., vol. 8 (6), August, 1990.

2. C.A. Barckett, ed., "Special issue on light wave systems and components," IEEE Commun. Mag., 27 (10), October, 1989.

3. C.A. Brackett, "Dense wavelength division multiplexing networks: Principle and applications," IEEE J. Select. Areas in Commun. Vol. 8, pp. 948-964, 1990.

4. Paul E. Green, Jr., "Fiber Optic Networks," Prentice Hall, Inc., 1993. For example, see page 136 describing the acousto-optic beam deflector having too large wavelength bandwidth to be interesting.

5. S.E. Harris, S.T.K. Nieh and R.S. Feigelson, "CaMoO4 electronically tunable optical filter," Appl. Phys. Lett. 17(8), 223-225, 1970.

6. S.E. Harris and R.W. Wallace, "Acousto-Optic tunable filter," J. Opt. Soc. Amer. 59 (6), 744-747, 1969.

7. I.C. Chang, "Tunable acousto-optic filters: An overview," Proc. SPIE, vol. 90, pp. 12-22, 1976.

8. I.C. Chang, "Acousto-Optic tunable filters," in the book Acousto-Optic Singal Processing, ed. by N.J. Berg and J. N. Lee, Marcel Dekker, Inc. New York.

9. K.W. Cheung, M.M. Choy, and H. Kobrinski, "Electronic wavelength tuning using acoustooptic tunable filter and broad continuous tuning range and narrow channel spacing," IEEE Photon. Tech. Lett. Vol. 1 (2), pp. 38-40, 1989. 
10. B. L. Heffner, D.A. Smith, J.E. Baran, A. Yi-Yan and K.W. Cheung, "Integrated-optic acoustically tunable infra-red optical filter," Electron. Lett. 24(25), 1562-1563, 1988.

11. D. A. Smith, J.E. Baran, J.J. Johnson, and K.W. Cheung, "Integrated-optic acousticallytunable filters for WDM networks," IEEE J. Selected Areas in Communications, 8(6), 1151$1158,1990$.

12. D. A. Smith, M.M. Choy, A. d'Alessandro, J. E. Baran, and A. W. Rajhel, "Cascaded acoustooptic/ Fiber Fabry-Perot filter with Finesse over 2000," IEEE Photon. Tech. Lett. Vol. 5 (2), pp. 189-191, 1993.

13. D. A. Smith, R.S. Chakravarthy, Z. Bao, J.E. Baran, J.L. Jackel, A. d'Alessandro, D.J. Fritz, S.H. Huang, X.Y. Zou, S.M. Huang, A.E. Willner and K.D. Li, "Evolution of the acousstooptic wavelength routing switch," J. Lightwave Tech. 14(6), 1005-1019, 1996.

14. D. A. Smith and J.J. Johnson, Appl. Phys. Lett. 61 (9), 1025-1027 (1992).

15. H. Herrmann, K. Schafer, and W. Sohler, Appl. Phys. Lett. 6 911), 1335, (1994).

16. D. Marcuse, IEEE J. Lightwave Technol. LT-5, 268 (1987).

17. G. Griffel and A. Yariv, IEEE J. of Quantum Electron. 27, 1115 (1991).

18. R. C. Alferness, T. L. Koch, L. L. Buhl, F. Storx, F. Heismann, and M.J.R. Martyak, Appl. Phys. Lett. 55, 2011 (1989).

19. J. W. Carnahan and B. A. Pelz, "Acousto-optic spectral manipulator," US Patent No. 5434666, Jul. 18, 1995.

20. J. B. Soole, A. Scherer, H. P. LeBlanc, R. Bhat, and M.A. Koza, "Spectrometer on chip: a monolithic WDM components," Conf. Record. Optical Fiber Commun. Conference, p. 123, February, 1992. 
Figure Captions:

Figure 1 Acousto-optic wavelength-selective devices: (a) an acousto-optic tunable filter (AOTF), (b) an acousto-optic spectrum analyzer (AOS) and (c) grating-assisted acousto-optic tunable filter (GA-AOTF).

Figure 2 Schematic experimental setup of the GA-AOTF.

Figure 3 Spectra of a Fabry-Perot laser diode obtained from (a) a conventional acousto-optic spectrum analyzer and (b) GA-AOTF. The GA-AOTF clearly resolves the Fabry-Perot laser lines which are separated by $1.2 \mathrm{~nm}$ that is beyond the resolution of commercially availabble AOTF's.

Figure 4 Scanning of a spectrum for various rf acoustic frequencies: (a) $35 \mathrm{MHz}$, (b) $40 \mathrm{MHz}$ and (c) $45 \mathrm{MHz}$. 


\begin{tabular}{|c|c|c|c|}
\hline & $\mathrm{AOTF}^{(2)}$ & $\mathrm{AOS}^{(b)}$ & GA-AOTF $^{(b)}$ \\
\hline $\begin{array}{l}\text { Period or Beat length } \\
\qquad \text { (in } \mu \mathrm{m})\end{array}$ & $\frac{\lambda}{|\Delta \mathrm{n}|}(=15.5)$ & $\Lambda_{\mathrm{AO}}=\frac{\mathrm{v}}{\mathrm{f}}(=17.6)$ & $\begin{array}{c}\mathrm{d}=1 / 1200 \mathrm{~mm} \\
(=0.83)\end{array}$ \\
\hline $\mathrm{N}=\frac{\mathrm{L}}{\text { Period or beat length }}$ & $\frac{|\Delta \mathrm{n}| \cdot \mathrm{L}}{\lambda}$ & $\frac{\mathrm{L}}{\Lambda_{\mathrm{AO}}}$ & $\frac{\mathrm{L}}{\mathrm{d}}$ \\
\hline $\mathrm{N}$ for $\mathrm{L}=1 \mathrm{~cm}$ & 645 & 570 & 12,000 \\
\hline $\begin{array}{l}\text { Spectral Bandwidth for } \\
\mathrm{L}=1 \mathrm{~cm}\left(\delta \lambda=\frac{\lambda}{N}, \text { in } \mathrm{nm}\right)\end{array}$ & 2.4 & 2.7 & 0.13 \\
\hline Tuning range (in $\mathrm{nm}$ ) & $\begin{array}{c}\lambda \cdot \frac{\Delta f}{f} \\
\left(1550 \cdot \frac{16 M}{175 M}=142\right)\end{array}$ & $\begin{array}{l}\lambda \cdot \frac{\Delta f}{f} \\
(=1100)\end{array}$ & $\begin{array}{l}\frac{\lambda \cdot \Delta f}{v} \cdot d=\lambda \cdot \frac{\Delta f}{f} \cdot \frac{d}{\Lambda_{A O}} \\
(=52)\end{array}$ \\
\hline
\end{tabular}

Table I. Comparison of the three acousto-optic tunable filters shown in Figure 1, the AOTF, the conventional AOS and the GA-AOTF. The following typical parameters are used at the wavelength of $1.55 \mu \mathrm{m}$.

(a) $\mathrm{LiNbO}_{3}$, slow shear mode $(\Delta \mathrm{n}=0.1, \mathrm{v}=3700 \mathrm{~m} / \mathrm{sec}, \mathrm{f}=175 \mathrm{MHz}, \Delta \mathrm{f}=16 \mathrm{MHz})$

(b) $\mathrm{TeO}_{2}$, slow shear mode $\left(\mathrm{v}=617 \mathrm{~m} / \mathrm{sec}, \mathrm{f}=35 \mathrm{MHz}, \Delta \mathrm{f}=25 \mathrm{MHz}, \Lambda_{\mathrm{AO}}=\mathrm{v} / \mathrm{f}=17.6 \mu \mathrm{m}\right)$ 


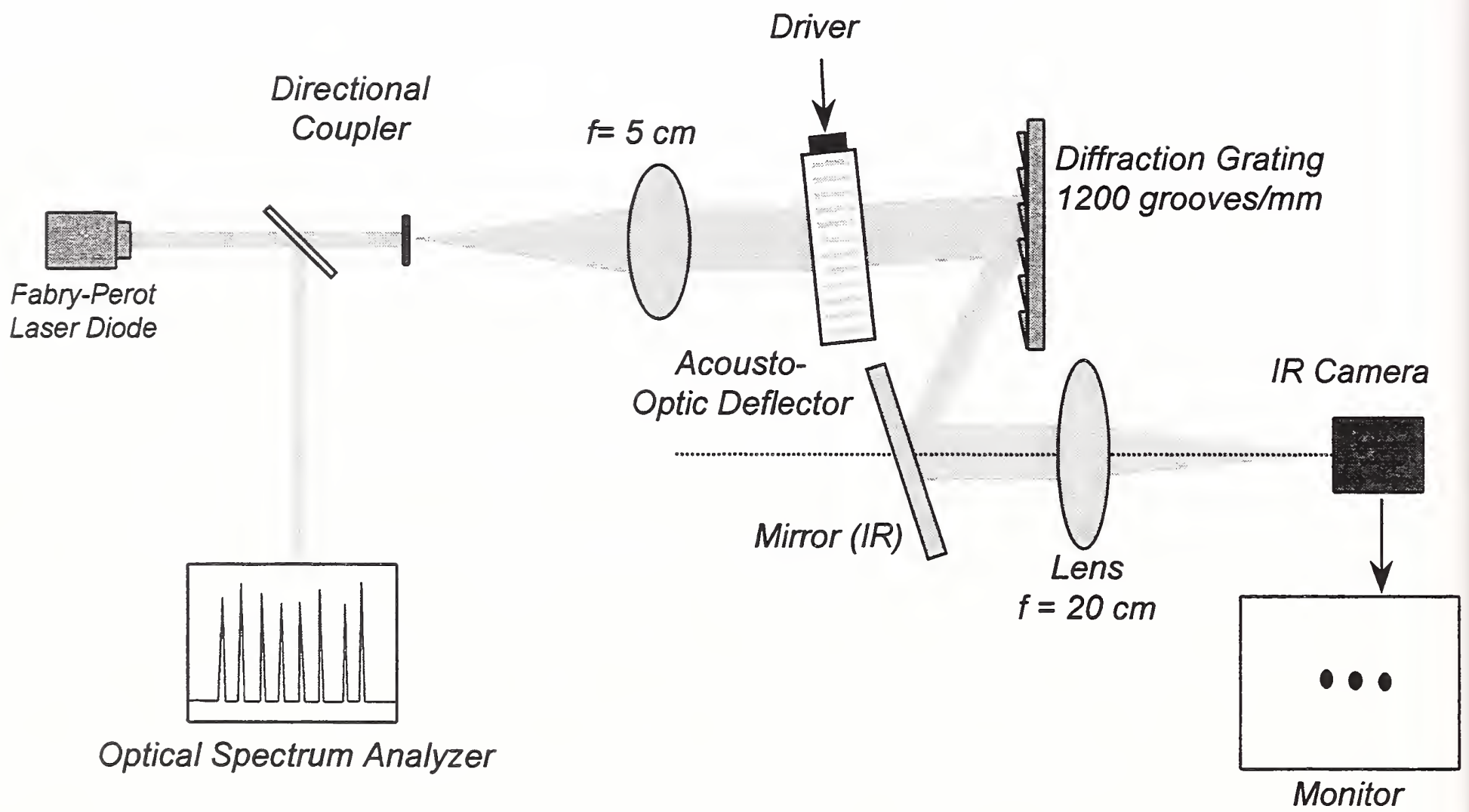




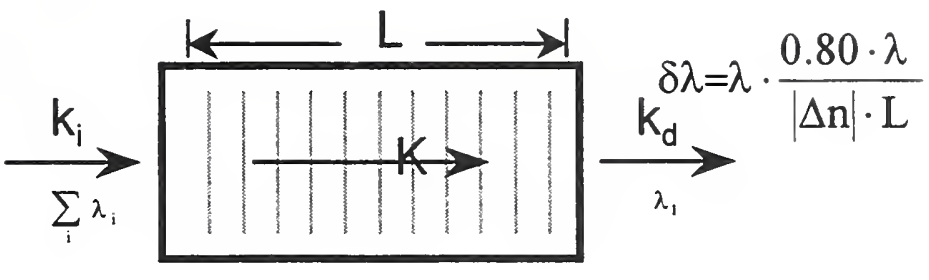

(a)

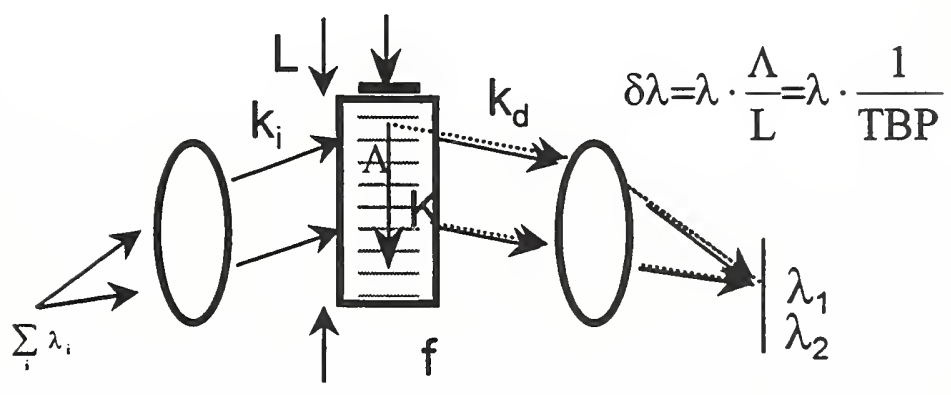

(b)

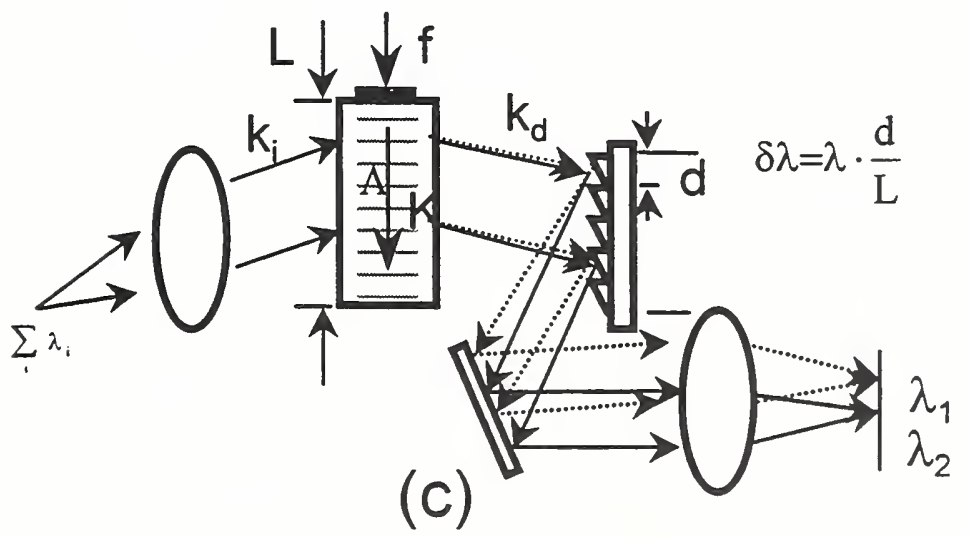




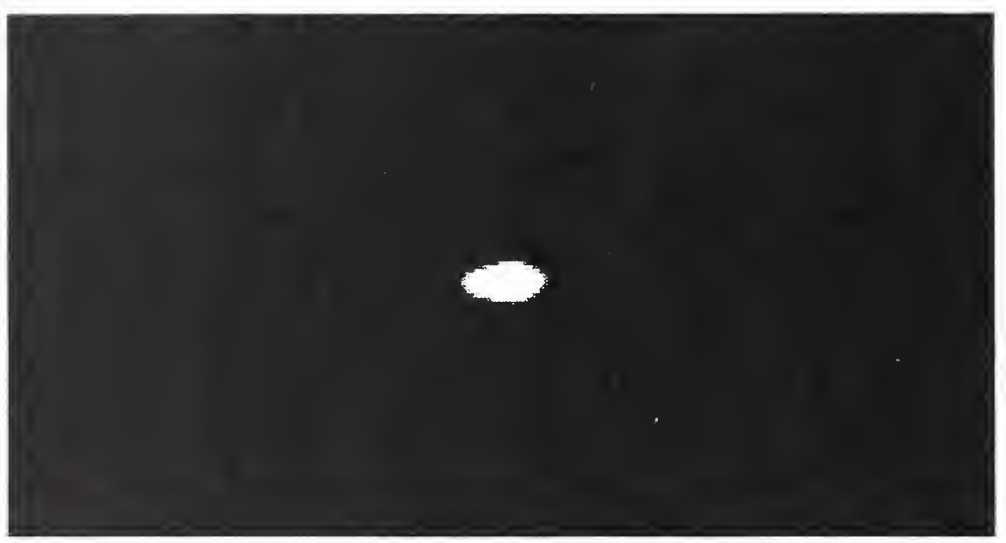

(a)

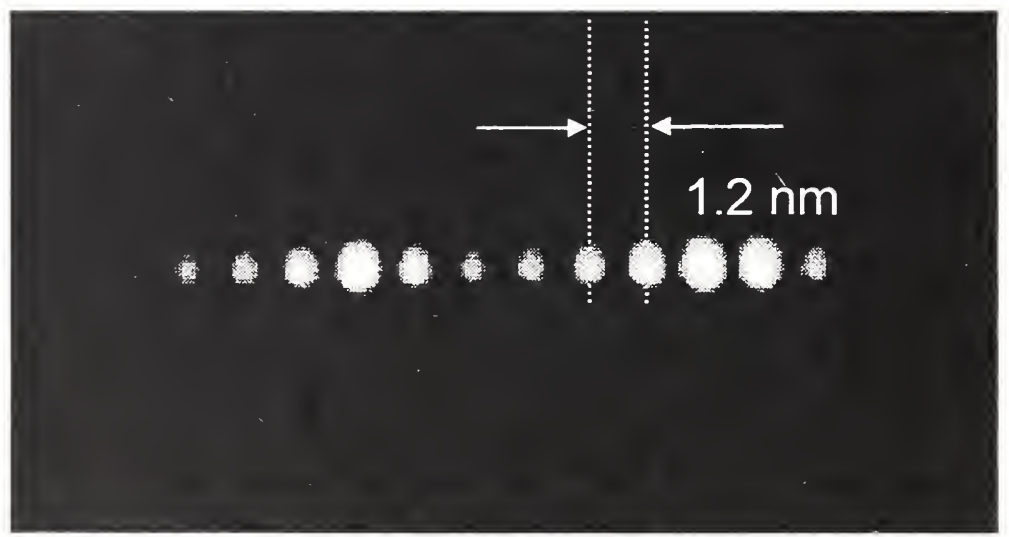

(b) 


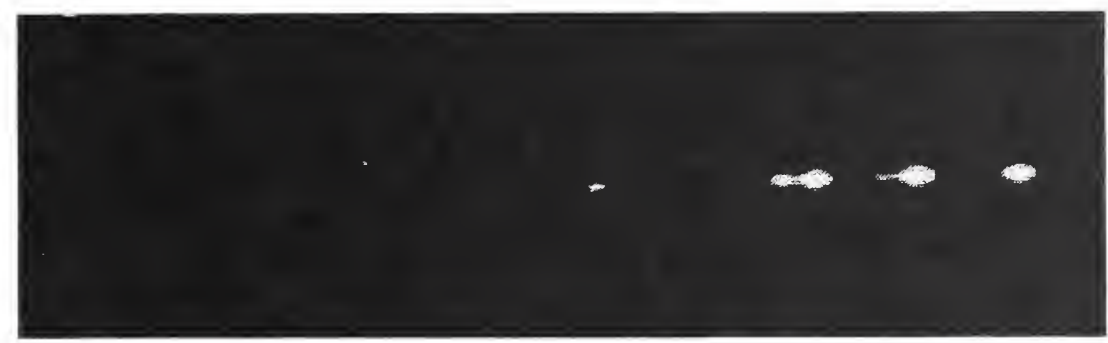

(a)

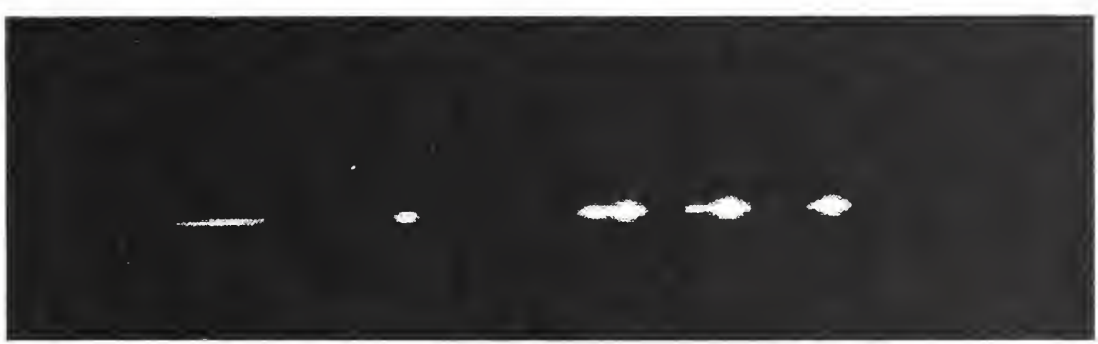

(b)

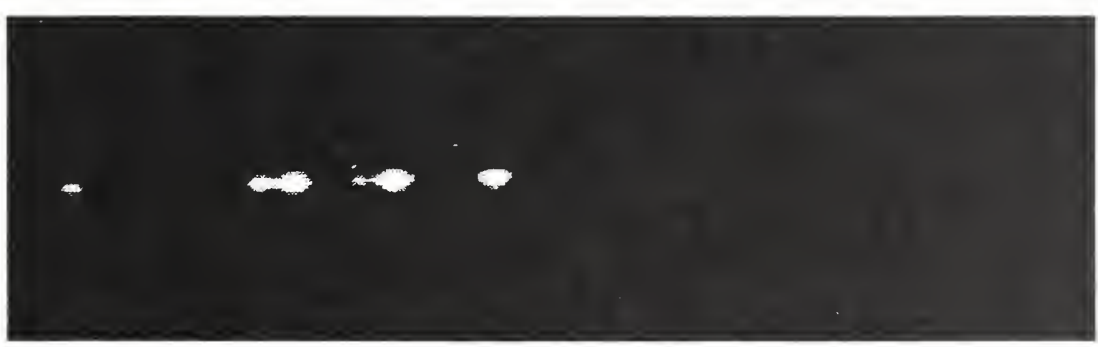

(c) 


